\title{
Convenios interinstitucionales como herramienta en la práctica tributaria. Beneficios e intencionalidad
}

\author{
Razo Ascázubi, Clara de las Mercedes* \\ https://orcid.org/0000-0002-6418-9549 \\ Chicaiza Taipe, Edgar Germánico* \\ https://orcid.org/0000-0001-7997-3363 \\ Paredes Vásquez, Jéssica Nataly* \\ https://orcid.org/0000-0002-3435-8057 \\ *Universidad Técnica de Cotopaxi, Latacunga, Ecuador \\ E-mail:clara.razo@utc.edu.ec \\ Recibido: 23 de diciembre de 2020 \\ Aprobado: 15 de enero de 2021
}

\section{Resumen}

El artículo presentado se centra en el análisis de la firma de convenio interinstitucional entre la UTC y el SRI, haciendo énfasis en el cumplimiento de los objetivos tanto para el estudiante como el contribuyente fiscal. En este contexto, su desarrollo se basa en la comprensión del alcance que este tipo de acuerdo puede llegar a tener y sus beneficios. Como primera instancia se analiza las características de naturaleza jurídica para luego evaluar los desafíos que debe enfrentar este convenio ya en la práctica tributaria; también se integra una valoración de los alcances en pro de un mejoramiento institucional y efectivo cumplimiento tanto del convenio como de recaudación de tributos. El diagnóstico se lo realiza en base a una investigación y análisis previo del convenio entre la Universidad Técnica de Cotopaxi (UTC) y el Servicio de Rentas Internas (SRI), estos datos se los recoge a través de una metodología cuantitativa, por lo que la información presentada es una recopilación de fuentes secundarias sometidas a un análisis comparativo. Como conclusión se observa un cumplimiento efectivo de los objetivos descritos en el convenio y un nivel de satisfacción del cliente adecuado según parámetros evaluados.

Palabras clave: Convenio UTC - SRI, NAF, Práctica tributaria, Contribuyente fiscal. 


\title{
Interinstitutional agreements as a tool in tax practice. Benefits and intentionality
}

\begin{abstract}
The article presented focuses on the analysis of the signing of the inter-institutional agreement between the UTC and the SRI, emphasizing the fulfillment of the objectives for both the student and the taxpayer. In this context, its development is based on the understanding of the scope that this type of agreement can have and its benefits. As a first instance, the characteristics of a legal nature are analyzed in order to then evaluate the challenges that this agreement must face in tax practice; an assessment of the scope is also integrated in favor of an institutional improvement and effective compliance with both the agreement and the collection of taxes. The diagnosis is made based on a previous investigation and analysis of the agreement between the Technical University of Cotopaxi (UTC) and the Internal Revenue Service (SRI), these data are collected through a quantitative methodology, so the information presented is a compilation of secondary sources subjected to comparative analysis. As a conclusion, an effective fulfillment of the objectives described in the agreement and an adequate level of customer satisfaction according to evaluated parameters is observed.
\end{abstract}

Keywords: UTC Convention - SRI, NAF, Tax practice, Taxpayer.

\section{Introducción}

El artículo presentado pretende establecer un análisis de la eficacia del convenio interinstitucional entre la Universidad Técnica de Cotopaxi y el Servicio de Rentas Internas, por lo que responde a un objetivo claro que es medir como primera instancia que los estudiantes y docentes de la UTC obtengan los beneficios planteados, que la población contribuyente reciba asesoría tributaria gratuita y por último una evaluación de satisfacción del cliente tanto institucional como final, es decir si los fines del convenio se cumplieron a cabalidad.

La figura del convenio interinstitucional en el marco de la legislación ecuatoriana, es aquel instrumento de cooperación entre una institución $\mathrm{y}$ otras personas de derecho jurídico públicas o privadas, en el contexto de apoyo mutuo tanto en recursos como en fortalezas. Es decir, el convenio es un acuerdo de voluntades entre dos o más personas o instituciones. Oficina Central de Cooperación, (UNP, 2017)

Para ello el análisis realizado tiene dos posturas, la primera el plantear los principales elementos del convenio interinstitucional, y como segundo propósito, se busca evaluar los resultados obtenidos en los años de ejecución del acuerdo, poniendo en consideración los resultados obtenidos en la práctica frente a los beneficiarios tomando en cuenta los principales desafíos que afronta el acuerdo interinstitucional.

Se parte argumentando que el convenio firmado entre la Universidad Técnica de Cotopaxi (UTC) y el Servicio de Rentas Internas (SRI), está basado en la creación del proyecto Núcleo de Apoyo Contable y Fiscal (NAF), siendo la finalidad de éste ser un centro gratuito de difusión del conocimiento contable y tributario, enfocado a instruir a los contribuyentes no obligados a llevar contabilidad y microempresarios la importancia en el cumplimiento de sus deberes formales. (Gallo, 2018)

El Servicio de Rentas Internas (SRI) nace en el año 1997, como respuesta a la evasión tributaria presentada en aquella época, así como una limitada cultura tributaria, por lo que la institución define políticas y estrategias de gestión que mejore la recaudación de impuestos. En los últimos años se evidencia crecimiento en el cobro de tributos, así como la eficiencia administrativa institucional. Los esfuerzos en gestión para mejorar los procesos se observaron en resultados de recaudación, tomando en consideración que la recaudación del

Razo, Clara; Chicaiza, Edgar y Paredes, Jéssica 
periodo comprendido entre 2007 y 2013 ascendió a 60.000 millones de dólares; éste resultado se ha enfocado en la aplicación de mejoras digitales e implementación de sistemas de alta tecnología, así como el incremento significativo de contribuyentes. SRI, (2020)

En el año 2014 se firma el convenio de Cooperación entre la UTC - SRI No 256 "SRI" y "LA UNIVERSIDAD" donde se da inicio con los NAF, entregando información tributaria y contable de forma gratuita a personas naturales no obligadas a llevar contabilidad y microempresarios.

Los beneficiarios directos de la aplicación de este convenio son los contribuyentes tributarios, ya que se enfoca en prestar asesoría de forma gratuita en el tema de declaraciones de impuestos evitando así el pago de multas y sanciones a la Administración Tributaria. Por otra parte, los estudiantes de la Universidad Técnica de Cotopaxi específicamente de la Carrera de Contabilidad y Auditoría, que son aquellos que tienen la oportunidad de poner en práctica los conocimientos adquiridos durante su formación académica sin que esto represente un riesgo profesional, así como la posibilidad de contar con mentores durante la ejecución del convenio.

Estos beneficios y beneficiarios, se vuelven un hito de partida como una expectativa para la ejecución del acuerdo entre instituciones, por lo que se estima una valoración real ya en la aplicación para establecer logros y resultados.

\section{Definiciones}

En éste apartado se pone a consideración los elementos que determinan a los convenios, y las características que merecen ser estudiados para la comprensión lectora.

Para ello se cita que el convenio tiene su naturaleza jurídica, lo que implica que debe ser constituido como un acuerdo de voluntades entre las partes, siendo estos sujetos de derecho o entidades legalmente competentes o autorizadas normativamente, en función de su respectiva formación legal, tomando en cuenta que estas instancias compartan un objetivo común. (Mezarina \& Rosales, 2019)

Es necesario que las instituciones que administrativa y jurídicamente coincidan en la voluntad de generarse obligaciones entre sí, estén vinculadas en el caso de hacer efectivo el cumplimiento de dicho instrumento mostrando el interés de actuar institucionalmente.

Los NAF se constituyeron como centros de información tributaria y contable, para entregar a los contribuyentes atención gratuita, con la finalidad de facilitar los procesos tributarios y generar una mejor cultura, este centro también busca dar apoyo y capacitación a estudiantes y docentes universitarios.

Los servicios ofrecidos por los NAF se especifican a continuación (Gallo, 2018):

- Información general contable y para la presentación oportuna de obligaciones y trámites tributarios (RUC, facturación, declaraciones y anexos, vehículos, etc.)

- Información general sobre obligaciones tributarias pendientes.

- Información general sobre el Régimen Impositivo Simplificado (RISE).

- Información general sobre medios de pago de impuestos y deudas tributarias.

- Información general sobre devolución de impuestos a Adultos Mayores y Discapacitados.

- Información general para la utilización de los servicios en línea del SRI.

En cuanto a la conceptualización de la práctica tributaria, se debe comprender que su naturaleza se centra en la cultura para el acatamiento de las reglas en el cumplimiento de las obligaciones tributarias, lo que quiere decir, respetar los elementos y procesos propuestos por el organismo rector.

Para esto se ha establecido en la Ley de Régimen Tributario Interno los factores de tributación y el SRI los procesos de aplicación de la norma, buscando tener una mayor efectividad en la recaudación, iniciando con programas para impulsar una cultura 
tributaria y por otro lado el cumplimiento a cabalidad en la recaudación de los tributos.

De acuerdo a lo estipulado en el Código Tributario en el Art. 15, las obligaciones tributarias se consideran un vínculo jurídico personal, entre el Estado o las entidades acreedoras de tributos y los contribuyentes o responsables de aquellos. (Función Legislativa, 2016)

La evasión tributaria, tiene el alcance desde la omisión de la presentación de la declaración de impuestos o la ausencia de pago de los impuestos generados, así como la disminución adulterada de los valores con los que serán calculados dichos tributos. (Pita, 2007)

En el caso de los beneficiarios se define como aquellos actores que participan activamente de las actividades del programa y perciben el beneficio económico. (Mancini, 2015) Para el caso de estudio se especifican como beneficiarios directos a las personas naturales no obligadas a llevar contabilidad, sociedades y estudiantes de la Carrera de Contabilidad y Auditoría de la Universidad Técnica de Cotopaxi.

Se incluye también en el estudio conceptual el análisis de satisfacción al cliente en el que se basa los resultados de la ejecución del convenio, partiendo de que la satisfacción del cliente es estudiada en diversas disciplinas y desde distintos enfoques, como lo cita (Quispe y Ayaviri, 2016) en su artículo, sociología (Campbel, 1987), psicología (Quintanilla, 1994) político y otros (Scherer, 1980). Sin embargo, la satisfacción del cliente se ha estudiado como respuesta a un estímulo dado, que puede ser evaluado en el momento del consumo o cuando hay reclamos (Howard, 1993). En consecuencia, la satisfacción del cliente puede interpretarse desde varios puntos de vista, en este caso desde el cumplimiento de las actividades programadas en el convenio tanto para los estudiantes en la formación tributaria y los contribuyentes en una asesoría que se brinde para evitar errores en declaraciones, omisiones y evasión involuntaria en la declaración de tributos.

\section{Metodología}

Para el estudio de caso se plantea una metodología basada en determinar los resultados concluyentes de la aplicación del convenio realizado entre la UTC y el SRI, este diseño se enfoca en el levantamiento de datos exactos frente a las situaciones planteadas como elementos fundamentales para la ejecución del convenio. Es por esto que se traza una investigación en base a los beneficiarios con la finalidad de determinar qué actividades si fueron desarrolladas frente al análisis de la conducta del contribuyente. Considerando estos elementos se establece como punto de partida el enfoque cuantitativo que permita hacer una valoración exacta sobre los factores que contempla el convenio. También se realiza un análisis documental basado en fuentes secundarias de un estudio preliminar realizado por (Gallo, W. 2018).

También se muestra en este artículo una recopilación bibliográfica, utilizando una revisión transversal en textos relacionados con el tema; así como en artículos de impacto científico orientados a complementar el estudio que garanticen el rigor de los resultados.

El estudio abarca tres etapas, iniciando en un análisis documental, para luego determinar mediante datos secundarios el estado base del convenio para luego concluir con una investigación descriptiva mostrando los resultados del convenio.

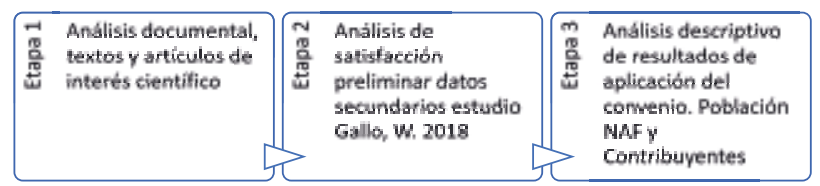

Figura 1. Metodología de la Investigación

Fuente: Investigación, Razo C. 2019

Los datos preliminares analizados corresponden a un estudio realizado en el año 2018, donde el autor (Gallo, 2018), plantea un estudio a las personas naturales con obligaciones tributarias en la ciudad de Latacunga para verificar el cumplimiento de los deberes formales. Estos datos no solo fueron levantados a través de la contribución de los beneficiarios sino de todos los involucrados en dicho convenio, obteniendo una visión más clara. El autor

Razo, Clara; Chicaiza, Edgar y Paredes, Jéssica 
cita que en el año 2017 se atendió aproximadamente a 8.715 usuarios, siendo la base poblacional para el estudio.

Los beneficiarios investigados corresponden a:

- Contribuyentes no obligados a llevar contabilidad de la Provincia de Cotopaxi.

- Sociedades, quienes requieran de los servicios que brindan los estudiantes de la (UTC) en convenio con el SRI.

- Estudiantes de la Carrera de Contabilidad y Auditoría de la Universidad Técnica de Cotopaxi.Servicio de Rentas Internas

\section{Principales desafíos y logros del convenio interinstitucional}

\subsection{Desafíos del Convenio}

La Universidad, al firmar el convenio con el Servicio de Rentas Internas ha buscado afianzar la relación institucional e impulsar el conocimiento de su comunidad estudiantil, por lo que es importante recalcar que el desafío institucional está en el cumplimiento del objetivo del mismo documento, que se centra en la asesoría tributaria para los usuarios que acuden a esta entidad de control.

Este acuerdo permite que exista una interacción entre el usuario y los estudiantes, dotándolos de un valor agregado dado en el conocimiento, tanto los estudiantes como los docentes parten de una actividad de aprendizaje previa para luego continuar con el soporte técnico a los contribuyentes que presentan problemas en el manejo de la plataforma para el cumplimiento de las obligaciones tributarias.

La visión expuesta por los docentes es que el convenio busca contar con centro gratuito para difundir el conocimiento contable y tributario, en este sentido que los estudiantes logren poner en práctica lo aprendido en su formación profesional, el contacto con el cliente y la solución de problemas reales.

\subsection{Hallazgos en el periodo de evaluación}

El Núcleo de apoyo Contable y Fiscal atendió 8.715 usuarios, donde su enfoque fue ayudar con asesoría para solventar inquietudes en cuanto al cumplimiento de sus obligaciones tributarias.

Como punto de partida en el análisis, se realizó una evaluación de la satisfacción de la atención recibida en el NAF a través de encuestas aplicadas a usuarios y sometidas a la fórmula del nivel de satisfacción propuesta en el estudio de (Gallo, 2018) como se muestra en la figura 2.

Nivel de Satisfacción $=$ Número de Usuarios Muy Satisfechos atención NAF Total de Usuarios Atendidos en el NAF

Figura 2. Fórmula de Satisfacción al Cliente Fuente: (Gallo, 2018), Adaptación Razo, 2019

Como se planteó inicialmente uno de los objetivos de evaluación es la valoración de la satisfacción al cliente o usuario final, por lo que se realizó en el NAF una investigación sobre los servicios recibidos a los contribuyentes atendidos; siendo el resultado de éste indicador de nivel de satisfacción del 94\%, lo que significa que 8.204 usuarios se encuentran muy satisfechos, de acuerdo a la fórmula de evaluación propuesta. Ésta valoración pone en evidencia que los servicios tributarios proporcionados por estudiantes y docentes se ajustan a las necesidades de los clientes requieren mejorando su situación tributaria.

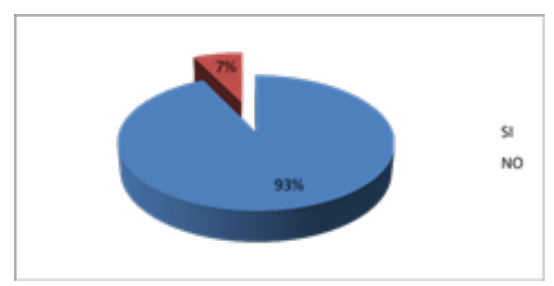

Figura 3. Capacitación estudiantil

Fuente: (Gallo, 2018), Adaptación Razo, 2019

En el caso del cumplimiento en el objetivo de la capacitación acordada en el convenio para estudiantes y docentes, se presenta un resultado del $93 \%$ en el que de acuerdo a la investigación se cumple, lo que quiere decir que el organismo rector en temas tributarios presta información oportuna y relevante para la ejecución de procesos tanto legales como operativos. 
Estos dos factores presentados son dos pilares fundamentales en la ejecución del convenio ya que muestra, por una parte, el cumplimiento del acuerdo de capacitación y por otro lado la eficiencia en la ejecución de los procedimientos adquiridos en la formación profesional y en la interacción institucional.

\subsection{Cumplimiento en la ejecución del Convenio}

Si se parte de los factores que integran el convenio es primordial realizar una valoración estadística concluyente, donde se analice indicadores porcentuales de cumplimiento, en este caso se planteó como resultados relevantes el cumplimiento de los procesos de capacitación, la supervisión permanente del docente de apoyo, las instalaciones del NAF donde se atiende al usuario, los eventos de promoción y difusión para estudiantes y el seguimiento al proyecto por parte de la institución educativa y que el SRI asigne a un responsable de dar cumplimiento al convenio.

Los resultados estadísticos del estudio realizado en el año 2018 se muestran en la tabla 1, donde se puede valorar que los ítems pactados en el acuerdo se han cumplido a cabalidad por parte de las dos instituciones.

Tabla 1

Evaluación estudiantil sobre elementos de convenio

\begin{tabular}{ll}
\hline $\begin{array}{l}\text { Objetivos del Convenio con } \\
\text { estudiantes }\end{array}$ & Cumplimiento \\
\hline $\begin{array}{l}\text { Cumplimiento de capacitación } \\
\text { Supervisión docente }\end{array}$ & $93 \%$ \\
Instalaciones NAF & $89 \%$ \\
$\begin{array}{l}\text { Eventos de difusión para promoción } \\
\text { el NAF }\end{array}$ & $100 \%$ \\
$\begin{array}{l}\text { Seguimiento } \\
\text { Fuente: Compilación estudio (Gallo, 2018), Adaptación } \\
\text { Razo, 2019 }\end{array}$ & $100 \%$
\end{tabular}

Siserealiza un recuento de los objetivos planteados en el convenio se puede concluir en función de los datos encontrados que, la capacitación ofrecida por el SRI como parte del acuerdo se cumple tanto para docentes como para los estudiantes, que los docentes de la UTC realizan su labor como supervisores del proceso dando un respaldo técnico, las instalaciones del NAF son buenas para la ejecución del proyecto y que el mismo tiene la promoción adecuada para que se integren en su ejecución los participantes. Es decir, que los objetivos específicos del convenio llegan a cumplirse en cuanto a ejecución.

Sin embargo, es importante también contrastar los resultados con la valoración que realiza el cliente final o beneficiario de este programa.

Tabla 2

Valoración a usuarios

Objetivos del Convenio con estudiantes Cumplimiento

Infraestructura

$86 \%$

Conocimientos en materia tributaria

$86 \%$

Supervisión

$95 \%$

Eventos de difusión para promoción del NAF

$92 \%$

Atención al cliente

$96 \%$

Fuente: Compilación estudio (Gallo, 2018), Adaptación Razo, 2019

En función de lo investigado, se puede determinar que la percepción del cliente es más baja que la que posee el estudiante, considerando que se requiere de mayor experticia y desenvolvimiento en el campo profesional. Cabe mencionar que en cuanto a la satisfacción del cliente en atención se mantiene la calificación en un nivel elevado. Sin embargo, se debe especificar que en ambos casos se cumplen los objetivos con los que se desarrolló el convenio, lo que quiere decir que si se realizan aportes significativos a la educación y ciudadanía respectivamente cuando se realizan acuerdos interinstitucionales.

\subsection{Toma de decisiones frente a la ejecución del convenio}

La ejecución del convenio de acuerdo a los datos presentados se ha cumplido, sin embargo, se

Razo, Clara; Chicaiza, Edgar y Paredes, Jéssica 
observa indicadores que corregir ya que si se realiza una evaluación de efectividad deberá plantearse otros factores referentes a la calificación y ejecución de resultados. En este caso se ha presentado una evaluación de cumplimiento con los objetivos propuestos inicialmente en el convenio, con lo que se puede decir que es satisfactorio, poniendo en contexto los resultados se establece que el convenio contribuye al mejor desempeño en cuanto a la declaración de tributos como se puede observar en la tabla 3 en función de los usuarios atendidos por caso.

Tabla 3

Concentración de casos atendidos

\begin{tabular}{cccc}
\hline $\begin{array}{c}\text { IVA } \\
\text { MENSUAL }\end{array}$ & $\begin{array}{c}\text { SEMESTRAL } \\
\text { IVA }\end{array}$ & $\begin{array}{c}\text { IMPUESTO A LA } \\
\text { RENTA }\end{array}$ & $\begin{array}{c}\text { TOTAL USUARIOS } \\
\text { ATENDIDOS }\end{array}$ \\
\hline 4693 & 1455 & 1006 & 8715 \\
\hline
\end{tabular}

Fuente: NAF 2018, Adaptación Razo, 2019

Como se puede observar en la tabla 3 en el NAF se atienden varios casos de recaudación tributaria lo que contribuye a reducir la evasión como principio de gestión institucional propuesto por el SRI, es decir que además del cumplimiento del convenio y sus objetivos de capacitación estudiantil se cumplen otras acciones transversales que contribuyen no solo a la población estudiantil en su preparación profesional, si no que se transforma en un soporte técnico para la comunidad que no dispone de recursos para una asesoría privada de declaración de tributos.

\section{Discusión}

En función del estudio realizado se define varios conceptos y principios, el cumplimiento del convenio parte en la ejecución de los acuerdos previamente pactados, pero se debe especificar que no se define un estándar de calidad donde se pueda evaluar realmente la capacidad de ejecución de los participantes. Cabe recalcar que si el análisis se centra en la finalidad textual del convenio el cumplimiento sería óptimo, y como primera instancia se debería considerar como logros, pero si se quiere dar el segundo paso para el crecimiento y desarrollo se pretendería ampliar el contexto citado en el documento o implementar una normativa en el cumplimiento orientado más a resultados cuantitativos.

\section{Conclusión}

El cumplimiento de un convenio interinstitucional viene dado por una serie de elementos, partiendo principalmente de los pactados en la firma y discusión inicial del mismo. Este tipo de documento más allá de ser una formalidad se transforma en un documento de control de ejecución de las partes, es por ello que es de suma importancia destacar el alcance y los beneficiarios del mismo.

Otro de los factores fundamentales detectados es la ambigüedad que puede generarse en este tipo de acuerdos ya que el mero cumplimiento de los objetivos generales no puede ser la totalidad o el fin, por lo que es relevante ir integrando avances o necesidades en función de los requerimientos detectadas por los usuarios a quien se les considerará beneficiarios.

Finalmente, es de gran importancia realizar evaluaciones constantes, ver avances y supervisar todos los procedimientos realizados ya que eso permitirá que el convenio se extienda y logre desarrollarse a la par de los usuarios y de los estudiantes inmersos en este tipo de proyectos,

\section{Referencias bibliográficas}

Barragán L. y Camargo, C. (2019) Revista Voces y Silencios. Volumen 11. Número 1. Fortalecimiento de la calidad educativa a partir de los convenios de cooperación interinstitucional: un caso desde la educación artística.

Cadena, P., Rendón, R., Aguilar, J., Salinas, E., de la Cruz, F y Sangerman, D. (2017). Métodos cuantitativos, métodos cualitativos o su combinación en la investigación: un acercamiento en las ciencias sociales. Revista Mexicana de Ciencias Agrícolas. 8(7), 16031617.

Función Legislativa. (2016). Código Tributario. 
Quito: Registro Oficial 744, 29-IV-2016).

Cotopaxi, C. d. (13 de Marzo de 2014). Convenio de cooperación interinstitucional entre el servicio de rentas internas y la universidad técnica de Cotopaxi. Convenio de cooperación interinstitucional entre el servicio de rentas internas y la universidad técnica de Cotopaxi. Latacunga, Cotopaxi, Ecuador.

Mancini，I. (2015) http://www.scielo.org.co/pdf/ esju/v18n1/v18n1a04.pdf. Clasificación y producción de beneficiarios en una política de prevención social del delito

Mezarina, S., Rosales P. (2019) http://www. scielo.org.pe/pdf/derecho/n82/a13n82.pdf Los acuerdos interinstitucionales en la práctica peruana de derecho internacional

Pita, C. (2007) El intercambio de informaciones tributarias como instrumento de contención de la competencia desleal a nivel internacional. https://www.cepal.org/ilpes/noticias/ paginas $/ 2 / 27472 /$ Claudino $\% 20$ pita $\% 20$ INTERCAMBIO \%20 DE \%2 0INFO $\% 20$ TRIBUTARIA.pdf.

Quispe, G y Ayaviri (2016) https://www.uv.mx/ iiesca/files/2016/11/15CA201601.pdfMedición de la satisfacción del cliente en organizaciones no lucrativas de cooperación al desarrollo

SRI. (s.f.). Núcleos de Apoyo Contable. Ecuador. sri-mantiene-una-recaudacion-alta-los-impuestos. (s.f.). sri-mantiene-una-recaudacion-alta- losimpuestos. Obtenido de sri-mantiene-unarecaudacion-alta-los-impuestos: sri- mantieneuna-recaudacion-alta-los-impuestos

Staruss, A. \& Corbin, J. (2014). Bases de la investigación cualitativa (2. ${ }^{\mathrm{a}}$ ed). Editorial Universidad de Antoquia. 